\title{
Effect of maternal supplement beverage with and without probiotics during pregnancy and lactation on maternal and infant health: a randomized controlled trial in the Philippines
}

\author{
Jacinto Mantaring ${ }^{1}$, Jalil Benyacoub ${ }^{2^{*}}$, Raul Destura ${ }^{3}$, Sophie Pecquet ${ }^{4}$, Karine Vidal $^{2}$, Sheri Volger ${ }^{5}$
} and Valerie Guinto ${ }^{6}$

\begin{abstract}
Background: Adequate nutrition is essential during pregnancy and lactation to provide sufficient energy and nutrients to meet the nutritional requirements of the mother, fetus and infant. The primary objective of this study was to assess the effect of a maternal nutritional supplement enriched with probiotics during pregnancy and early lactation on the incidence of infant diarrhea.

Methods: Healthy, pregnant (24-28 weeks gestation) women were randomized 1:1:1 to receive either no supplement or two servings per day of an oral supplement (140 kcal/serving) providing $7.9 \mathrm{~g}$ protein, multivitamin/ minerals, and enriched or not with the probiotics Lactobacillus rhamnosus and Bifidobacterium lactis, from the third trimester of pregnancy until at least 2 months post-delivery. Incidence of infant diarrhea until 12 months postdelivery was analyzed by Poisson regression. The effect on maternal health, fetal growth, and infant growth and morbidity were also evaluated and analyzed by ANOVA.

Results: A total of 208 mother/infant pairs were included in the analysis. No significant difference in the incidence of infant diarrhea was observed between the three study groups. The mean maternal weight gains at delivery were similar among groups, despite an increase in caloric intake in the supplemented groups. No statistically significant differences between groups were observed in incidence of pregnancy-related or fetal adverse outcomes. Mean weight-, length-, BMI- and head circumference-for-age z-scores were below the WHO median value for all groups. Post-hoc analysis to compare the effect of the combined supplement groups versus the no supplement group on infant growth parameters showed, at 12 months, that the combined supplemented group had gained statistically significant more weight ( $8.97 \mathrm{vs} .8 .61 \mathrm{~kg}, p=0.001$ ) and height (74.2 vs. $73.4 \mathrm{~cm}, p=0.031)$, and had a higher weight-for-age $z$-score $(-0.62$ vs. $-0.88, p=0.045)$ than the no supplement group.
\end{abstract}

Conclusions: Maternal nutritional supplement with or without probiotics given during late pregnancy and early lactation was well tolerated and safe. Even though no difference in incidence of infant diarrhea was observed between the three groups, the analysis of the combined supplemented groups showed beneficial effects of maternal supplementation on infant weight and length gains at 12 months.

Trial registration: ClinicalTrial.gov: NCT01073033. Registered 17.02.2010.

Keywords: Maternal supplementation, Probiotics, Lactobacillus rhamnosus, Bifidobacterium lactis, Diarrhea, Safety, Infant growth

\footnotetext{
* Correspondence: jalil.benyacoub@rdls.nestle.com

${ }^{2}$ Institute of Nutritional Science, Nestlé Research Center, Vers-chez-les-Blanc,

1000, 26 Lausanne, Switzerland

Full list of author information is available at the end of the article
}

(c) The Author(s). 2018 Open Access This article is distributed under the terms of the Creative Commons Attribution 4.0 International License (http://creativecommons.org/licenses/by/4.0/), which permits unrestricted use, distribution, and 


\section{Background}

There is a substantial body of evidence showing that maternal diet throughout pregnancy and during breastfeeding could influence the infant growth, development and health $[1,2]$. Only a modest increase in energy intake is required during pregnancy compared to pre-pregnancy requirements. Indeed, approximately 100 and 300 additional kcal per day are needed in the first and second/third trimesters, respectively, with increased requirements during breastfeeding of $450 \mathrm{kcal}$ per day [3]. Conversely, the recommended intake during pregnancy for several nutrients such as iron, folate and vitamin B6 are up to 50\% higher than those for a non-pregnant state, which could prove challenging [3]. Geographic and sociodemographic differences have been observed regarding nutrient deficiencies in pregnancy. The World Health Organization (WHO) has warned that some nutrient deficiencies (especially for vitamin $\mathrm{A}$, iodine, vitamin $\mathrm{D}$, calcium and zinc) are more common in pregnant women in low resource countries compared to those with higher resources. Other micronutrients (i.e., vitamin B12, iron, and folate) are commonly deficient worldwide [3]. This is of particular importance considering that maternal undernutrition can lead to miscarriage and major developmental issues such as intrauterine growth restriction, low birth weight and premature births $[4,5]$. As many as 800,000 neonatal deaths per year are from small for gestational age births, many of which are attributed to maternal undernutrition [6]. It was estimated that stunting, wasting, and micronutrient deficiencies account for almost 3.1 million child deaths annually [6]. Ultimately, maternal nutrition, especially micronutrient intake, is an important determinant of health as an adult. This has been shown to have an impact on the development of cardiovascular diseases and obesity [7, 8].

In developing countries, with a high risk of under nutrition, the use of nutritional supplements during pregnancy is encouraged to ensure adequate supply of nutrients for both mother and fetus [5]. In a recent Cochrane review, Haider and Bhutta evaluated the benefits of oral multiple-micronutrient supplementation during pregnancy on maternal, fetal and infant health outcomes. The analysis of 15 clinical trials conducted in low and middle-income countries revealed that multiple-micronutrient supplementation with iron and folic acid resulted in a significant decrease in the number of low birth weight or small-for-gestational age newborn infants, and a reduced rate of stillbirth [9].

Dramatic immune and physiological changes take place during pregnancy to accommodate the growing fetus; these include alterations in the gut and vaginal microbiome populations [10]. These changes may influence the maternal metabolic profile and may contribute to the metabolic and immunological health of the infant [10]. The composition of the microbiome is influenced by many factors, including diet and intake of nutritional bioactives such as probiotics [10]. Probiotics can regulate gut and vaginal microflora, and probiotic supplementation has been used in some trials as a strategy to influence pregnancy and infancy outcomes [10]. Maternal transfer of bacterial signals is possible during pregnancy and lactation [11]. This (as well as the mother's diet and microbiota) can influence newborn microbial colonization and microbiota establishment which in turn may have an impact on infant's short and long-term development parameters, including growth [11].

Prior to the initiation of our study, the safety and effect of a maternal nutritional supplement containing a mix of probiotics for pregnant and breastfeeding women on fetal and infant growth was only partially addressed. Safe use of probiotics has been documented. Indeed, infants born from mothers receiving Lactobacillus rhamnosus were shown to have a significantly reduced risk of developing atopic eczema during the first two [12] to seven [13] years of life and no adverse effects were reported $[12,13]$. Researchers also observed an improved gut microbiota in babies born from women receiving probiotics during pregnancy [14] or during pregnancy and lactation [15], and again, these studies did not report any adverse events $[14,15]$. Some probiotic strains have also been successfully used to improve the outcome of gastrointestinal diseases, in particular diarrhea [16, 17]. Diarrhea episodes are common infant infections and are a major health concern in pediatrics.

Among the probiotic strains, Bifidobacterium lactis and Lactobacillus rhamnosus have been reported to improve diarrheal outcomes in infants [18-23]. Therefore, we conducted this study to assess the effect of an oral maternal nutritional supplement formulated with these two probiotics on the incidence of diarrhea in infants from birth to one year, assessed as the primary endpoint of the trial. Secondary objectives included examining the effect of an oral maternal nutritional supplement on maternal health, fetal growth, and infant growth and morbidity up to one year of age. Due to the overall low incidence of diarrhea reported in this study, we conducted an additional post-hoc analysis. It evaluated the effect of the combined oral supplement groups (with and without probiotics) compared to the no supplement group on maternal health and fetal and infant growth.

\section{Methods}

Study design

A single center, randomized, double blind trial of three parallel-groups (supplement, supplement plus probiotics, and no supplement) was conducted at the Community Hospital in Muntinlupa City, Philippines, between April 2010 and September 2012. The study protocol was approved by the institution's Ethics Committee (University of 
the Philippines Manila - National Institutes of Health Ethics Review Board; protocol number NIH 2009-027). The study was conducted in accordance with the Helsinki Declaration and complied with Good Clinical Practices as laid out in the International Conference on Harmonization guidelines. Written informed consent was obtained from all study participants after the nature and possible consequences of the study had been fully explained. The primary objective of the trial was to examine the effect of an oral nutritional supplement containing a mix of probiotics Lactobacillus rhamnosus and Bifidobacterium lactis, taken during pregnancy and up to minimally 2 months post-delivery on the incidence of diarrhea in infants from birth to one year of age. The secondary objectives were (i) in mothers, to assess the effect of oral nutritional supplements on fetal growth and on maternal health (weight gain and pregnancy-related adverse events [AEs]) during the third trimester of pregnancy and up to delivery; and (ii) in infants, to investigate the impact of nutritional supplement beverages on infant growth and morbidity.

\section{Study population}

Healthy women at the beginning of the third trimester of pregnancy (24 to 28 weeks of gestation) who were willing to exclusively breastfeed for at least the first 2 months postpartum were recruited and enrolled in the study. The study protocol encouraged exclusive breastfeeding up to 6 months of age. The infants born to study participants were followed until one year of age. Pregnant women were excluded from the study if they had any of the following exclusion criteria: known allergy to cow's milk, known history of lactose intolerance, previous diagnosis of human immunodeficiency virus (HIV) and hepatitis B, multiple pregnancy, high risk pregnancy (pre-eclampsia, diabetes, etc.), current or past participation in another clinical trial during the last three months, consumption of pro- and/or prebiotics-containing food/supplement in the month before inclusion.

\section{Study supplements and blinding}

The supplement and supplement plus probiotics formulations consisted of proteins, carbohydrates, fats with vitamins and minerals in amounts intended for nutritional support during pregnancy. Each serving of $200 \mathrm{~mL}$ contained $140 \mathrm{kcal}$ energy, $7.9 \mathrm{~g}$ of proteins, $21 \mathrm{~g}$ of carbohydrates, and $3.5 \mathrm{~g}$ of fat. It also contained minerals and vitamins (Table 1 ). The supplement plus probiotics contained the mix of probiotics composed of $7 \times 10^{8} \mathrm{cfu}$ of Bifidobacterium lactis CNCC I-3446 and $7 \times 10^{8} \mathrm{cfu}$ of Lactobacillus rhamnosus CGMCC 1.3724, per serving. Both supplements were manufactured by the sponsor, Nestlé (Product technology center, Konolfingen, Switzerland). The two supplements were indistinguishable and were supplied as powder, in sachets, with instructions to prepare $35 \mathrm{~g}$ of
Table 1 Nutritional composition of the supplements

\begin{tabular}{|c|c|}
\hline Energy, kcal & 140 \\
\hline Fat, g & 3.50 \\
\hline Linoleic acid, mg & 73.2 \\
\hline Alpha-linoleic acid, mg & 80.9 \\
\hline Docosahexaenoic acid, mg & 43.8 \\
\hline Protein, g & 7.9 \\
\hline Carbohydrates, g & 21 \\
\hline \multicolumn{2}{|l|}{ Minerals, mg } \\
\hline Sodium & 87 \\
\hline Chloride & 205 \\
\hline Calcium & 254 \\
\hline Magnesium & 32 \\
\hline Iron & 7 \\
\hline Zinc & 2.6 \\
\hline \multicolumn{2}{|l|}{ Vitamins } \\
\hline Vitamin A, IU & 438 \\
\hline Vitamin D, IU & 35 \\
\hline Vitamin E, IU & 4.60 \\
\hline Vitamin C, IU & 19.6 \\
\hline Vitamin B1, $\mu \mathrm{g}$ & 217 \\
\hline Vitamin B6, $\mu \mathrm{g}$ & 263 \\
\hline Niacin, $\mu \mathrm{g}$ & 3045 \\
\hline Folic acid, $\mu \mathrm{g}$ & 125 \\
\hline Panthotenic acid, $\mu \mathrm{g}$ & 1295 \\
\hline Biotin, $\mu g$ & 9.80 \\
\hline
\end{tabular}

The composition of the supplement plus probiotics is identical to the supplement alone except for the addition of $L$. rhamnosus $\left(7 \times 10^{8} \mathrm{cfu}\right)$ and $B$. Lactis $\left(7 \times 10^{8} \mathrm{cfu}\right)$

powder in $200 \mathrm{ml}$ of water. Both products were blinded by the manufacturer. Thus, the identity of the specific products was blinded to the subjects, support staff and investigators. The reference group not taking the supplement was not blinded.

\section{Randomization}

An in-house computer program (TrialSys) was used to generate a randomization sequence to allocate expecting mothers to either the supplement, supplement plus probiotics, or no supplement groups. Eligible pregnant women were randomized into the three groups with a 1:1:1 ratio. The investigator accessed allocation numbers via a web-based application. Random allocation remained concealed until assigned by computer program and could not be predicted based on previous assignments.

\section{Trial procedure}

Each supplemented group received $2 \times 200 \mathrm{ml}$ serving per day of the allocated nutritional supplement beverage 
(to be taken in the morning and evening). The no supplement group did not receive any nutritional supplement beverage. For both supplemented groups, feeding with the assigned study product begun at 24-28 weeks gestation and continued for two months (minimum) after birth. Although optional, mothers were encouraged to continue for six months after birth. When the mother completely stopped breastfeeding, supplementation was also stopped. The infants continued in the study until one year of age.

A total of 11 visits (from screening visit 0 to last visit 10) took place during the study, the major visits are illustrated in Fig. 1. Before birth, expecting mothers attended a screening visit (at 24-28 weeks' gestation, visit 0) during which history and subjects' data were collected, inclusion/exclusion criteria were checked, health was assessed, and body weight measured. A fetal ultrasound was performed and women randomized to the supplemented groups started the supplements. At each visit, the physician inquired about the occurrence of AEs (especially nausea, vomiting, and diarrhea), assessed health, and measured body weight. At delivery (visit 3), the same information was recorded along with completion of a delivery record, including mode of delivery and gestational age. Mothers were followed and examined (for AEs and health assessments) up to 2 months post-delivery. At birth, infants were examined, anthropometric measurements were taken and AEs (any untoward birth defect) recorded. After birth, study investigators examined the infants at 2 weeks and 1, 2, 4, 6, 7, and 12 (visit 10) months of age and took anthropometric measurements, collected and reviewed parent/caregiver-reported 3-day feeding and gastrointestinal tolerance diaries, and assessed the occurrence of any AEs and in particular episodes and duration of diarrhea, since last visit.

\section{Outcome measures}

The primary outcome was the incidence of diarrhea in infants from birth to one year (total count of diarrhea per infant per year). The incidence and duration of diarrhea episode were clinically assessed by the pediatrician at each visit, starting 2 weeks post-delivery through 12 months of age. Diarrhea was defined as at least three or more watery stools in a $24-\mathrm{h}$ period. An episode of diarrhea was considered to have ended after two consecutive non-watery stools or no stools for $24 \mathrm{~h}$.

In mothers, secondary outcomes included health assessment (clinical examination, weight gain); documentation of infections (genito-urinary tract infection); and tolerance to study product (nausea, vomiting, diarrhea).

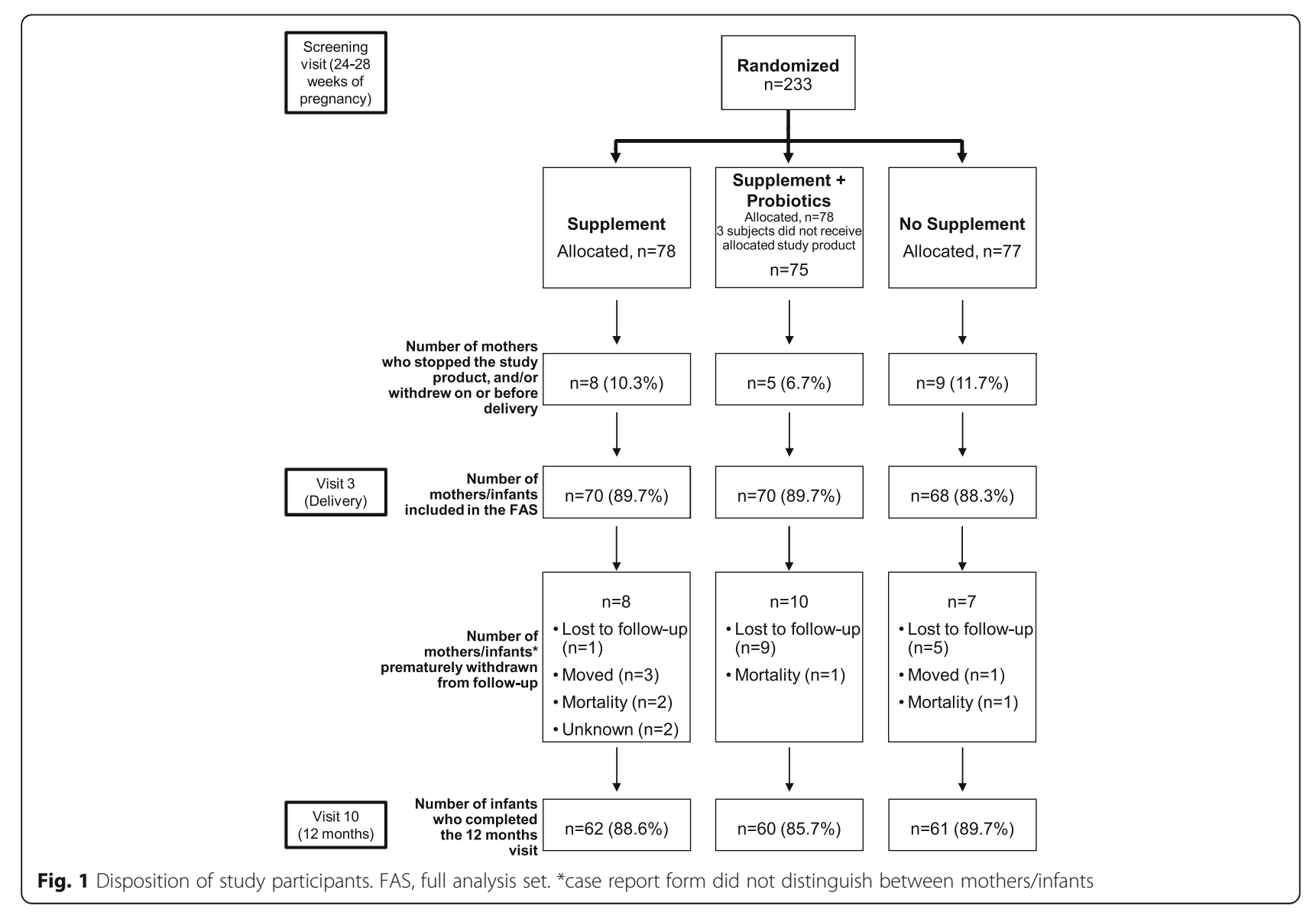


Fetal growth (abdominal circumference, biparietal diameter, and femoral length) was determined by ultrasound at enrolment and just before delivery.

In infants, secondary outcomes included anthropometry and morbidity. Anthropometry was measured by weight, recumbent length/height and head circumference at each visit from birth to 12 months. Infants were weighed unclothed to the nearest $10 \mathrm{~g}$ on the same electronic scales calibrated according to the manufacturer's instructions. Recumbent length was measured on a standardized length board to the nearest $1 \mathrm{~mm}$ with the body fully extended and feet flexed. Head circumference was measured using a standard plastic-coated measuring tape at approximately $2.5 \mathrm{~cm}$ above the eyebrows. Morbidity was inquired by the pediatrician at each visit and recorded in the AEs form.

\section{Adverse events and serious adverse events (SAEs)}

The study investigator evaluated the seriousness of all AEs and any potential relation to the study products. AEs and SAEs were collected from randomization of the mothers up to the 12-month follow-up period, and coded using the WHO Adverse Reactions Terminology (WHO-ART). A serious AE was defined as any untoward medical occurrence that at any dose: results in death; is life-threatening; requires inpatient hospitalization or prolongation of existing hospitalization; results in persistent or significant disability/incapacity; or is a congenital anomaly/birth defect.

\section{Statistical methods}

The sample size was calculated based on the articles of Hengstermann et al. [24] and Ortiz-Andrellucchi et al. [25]. Based on these two reference articles, a two sample test on proportion led to a number of 62 subjects per group in order to show a $50 \%$ decrease in incidence of diarrhea with a significance of 0.05 and a statistical power of $80 \%$. Considering an estimated $20 \%$ of dropouts, a total number of 234 infants (78 per randomized group) were planned to be recruited into the study, and a total of 62 infants per group were supposed to be seen at 1 year of age. Those numbers were achieved as indicated in Fig. 1. The primary outcome was the total count of diarrhea per infant per year. The incidence of diarrhea was analyzed by Poisson regression in the full analysis set (FAS). Secondary outcomes (anthropometric measurements and other continuous outcomes) were analyzed by ANOVA correcting for sex and available baseline, respectively. A post-hoc analysis comparing the combined supplemented groups (oral nutritional supplements with and without probiotics) versus the no supplement group was performed. The aim of this post-hoc analysis was to assess the following outcomes in infants: body weight gain up to one year of age (non-inferiority analysis); anthropometric measurements at 12 months of age (body weight, length, head circumference and body mass index $[\mathrm{BMI}]$ ); anthropometric $\mathrm{WHO}$ z-scores over time (weight-, length-, BMI- and head circumference-for-age); and infant's morbidity. All outcomes were analyzed both by per protocol and by intention to treat data sets.

\section{Results \\ Mothers and infants demographics and baseline characteristics}

A total of 233 expecting mothers attending a single community hospital were randomized to one of the three study groups. Twenty-five women were not exposed to the study supplement and/or withdrew from the study on or before delivery. The results described in this section are based on the FAS population. Two hundred and eight mothers and the same number of infants were included in the FAS: 70 in the supplement group, 70 in the supplement plus probiotics group, and 68 in the no supplement group. Eight, 10, and 7 mothers/infants were excluded from the FAS in the supplement, supplement plus probiotics, and no supplement groups, respectively. A total of 183 infants completed the 12 months study visit (Fig. 1).

The demographics and baseline characteristics of mothers and infants were similar across the three groups (Table 2). All infants were in good health at birth with similar APGAR scores measured at 1 and $5 \mathrm{~min}$ after birth. The mean body weights at birth were similar for infants from the two supplemented groups $(2.93 \mathrm{~kg}$ \pm 0.47 and $2.90 \mathrm{~kg} \pm 0.39$, supplement and supplement plus probiotics, respectively) and for the no supplement group $(2.88 \mathrm{~kg} \pm 0.44)$. At 4 months of age, $64.3 \%$ (supplement), $64.3 \%$ (supplement plus probiotics), and $72 \%$ (no supplement) of the infants were still on exclusive breastfeeding in their respective groups.

\section{Maternal supplementation during pregnancy Maternal and fetal anthropometrics during last trimester of pregnancy}

Maternal weight gain Pre-pregnancy weight and BMI were very similar across all study groups, reflecting a homogeneous study population (Table 3). Despite a daily increase in calorie intake of about $280 \mathrm{kcal}$ in the supplement and supplement plus probiotics groups, no significant difference in median maternal weight gain was observed between the supplemented groups and the no supplement group (supplement plus probiotics vs. no supplement, $p=0.945$; supplement vs. no supplement, $p=$ $0.892)$. Mean maternal weight gain at delivery was $10.6 \mathrm{~kg}$, $10.5 \mathrm{~kg}$, and $10.3 \mathrm{~kg}$, in the supplement, supplement plus probiotics, and no supplement groups, respectively. Mean BMI at delivery was similar among groups (Table 3).

Fetal growth and development Infants were born at approximately 39 weeks gestation with no statistically 
Table 2 Maternal and infants baseline characteristics

\begin{tabular}{|c|c|c|c|}
\hline Characteristics & Supplement & Supplement + probiotics & No supplement \\
\hline Mothers, $n$ & 70 & 70 & 68 \\
\hline Age at randomization in years, mean (SD) & $26.3(5.5)$ & $24.8(5.3)$ & $24.9(5.4)$ \\
\hline Ethnic origin, \% of Asian & 100 & 100 & 100 \\
\hline First-time mothers, $\%$ & 45.7 & 40.0 & 52.9 \\
\hline Type of delivery, \% C-section & 21.7 & 15.7 & 13.2 \\
\hline Education, \% of secondary education & 73.9 & 55.7 & 73.5 \\
\hline Infants at birth, $\mathrm{n}$ & 62 & 60 & 61 \\
\hline Gestation age at birth in weeks, mean (SD) & $38.8(1.5)$ & $38.8(2.0)$ & $38.8(1.2)$ \\
\hline Sex, \% of males & 50 & 62.9 & 44.1 \\
\hline APGAR score at 1 min, median (min-max) & $9.0(4.0-9.0)$ & $9.0(6.0-9.0)$ & $9.0(6.0-9.0)$ \\
\hline APGAR score at 5 min, median (min-max) & $9.0(8.0-9.0)$ & $9.0(5.0-10.0)$ & $9.0(9.0-9.0)$ \\
\hline Length in cm, mean (SD) & $50.1(2.7)$ & $49.9(2.8)$ & $49.7(2.4)$ \\
\hline Weight in kg, mean (SD) & $2.93(0.47)$ & $2.90(0.39)$ & $2.88(0.44)$ \\
\hline Body mass index in $\mathrm{kg} / \mathrm{m}^{2}$, mean (SD) & $11.6(1.3)$ & $11.6(1.3)$ & $11.6(1.2)$ \\
\hline Head circumference in cm, mean (SD) & $32.7(1.3)$ & $32.8(1.7)$ & $32.5(1.6)$ \\
\hline
\end{tabular}

SD Standard deviation

significant differences between the three groups in mean birth weight, APGAR scores, and anthropometrics (Table 2). Similarly, no significant differences were observed between the three groups for abdominal circumference (mean values of $32.64,32.29$, and $32.20 \mathrm{~cm}$, in the supplement, supplement plus probiotics, and no supplement groups, respectively), biparietal diameter (mean values of $8.85,8.86$, and $8.82 \mathrm{~cm}$ in the supplement, supplement plus probiotics, and no supplement groups, respectively), or femoral length (mean values of $6.88,6.75$, and $6.76 \mathrm{~cm}$, in the supplement, supplement plus probiotics, and no supplement groups, respectively) (Fig. 2). Overall, there were no statistically significant differences between the three groups for infant growth parameters during fetal development.

\section{Pregnancy-related AEs and SAEs}

There were no statistically significant differences in the number of pregnancy-related AEs and SAEs recorded during the study between the three groups (Table 4). None of the SAEs were considered as related to the study supplements.

\section{Maternal supplementation during lactation Infant outcome measures}

Incidence of diarrhea During the 12-month follow-up, $12.9 \%$ (9/70), $14.3 \%(10 / 70)$, and $16.2 \%(11 / 68)$ of infants experienced diarrhea in the supplement, supplement plus probiotics, and no supplement groups, respectively. One serious case of diarrhea was reported in the supplement group. There was no statistically significant difference in the incidence of diarrhea observed between the three groups. The incidence $(95 \%$ confidence interval [CI]) was $0.17(0.09-0.30)$ in the supplement group, 0.14 $(0.07-0.27)$ in the supplement plus probiotics group, and $0.19(0.11-0.33)$ in the no supplement group (supplement plus probiotics group vs. supplement group, $p=0.692$; supplement plus probiotics group vs. no supplement group, $p=0.509$ ). When supplemented groups were combined, the incidence (adjusted mean $[95 \% \mathrm{CI}]$ ) was $0.15(0.10-0.24)$ in the combined supplement groups vs. $0.19(0.11-0.33)$ in the no supplement group $(p=0.586)$. The mean product effect with combined

Table 3 Maternal weight status until delivery

\begin{tabular}{|c|c|c|c|}
\hline Mean (SD) & $\begin{array}{l}\text { Supplement } \\
\qquad(n=70)\end{array}$ & $\begin{array}{l}\text { Supplement + probiotics } \\
\qquad(n=70)\end{array}$ & $\begin{array}{l}\text { No supplement } \\
\qquad(n=68)\end{array}$ \\
\hline Declared pre-pregnancy weight (kg) & $49.1(8.2)$ & $49.2(7.7)$ & $49.1(7.7)$ \\
\hline Pre-pregnancy BMI $\left(\mathrm{kg} / \mathrm{m}^{2}\right)$ & $20.6(2.9)$ & $20.7(2.7)$ & $21.1(3.3)$ \\
\hline $\begin{array}{l}\text { Maternal weight gain from baseline (24-28 weeks of pregnancy) } \\
\text { up to delivery ( } \mathrm{kg} \text { ) }\end{array}$ & $5.00(2.69)$ & $4.55(2.25)$ & $4.62(2.16)$ \\
\hline Maternal weight gain at delivery ${ }^{\mathrm{a}}(\mathrm{kg})$ & $10.6(5.8)$ & $10.5(4.0)$ & $10.3(7.0)$ \\
\hline Maternal BMI at delivery $\left(\mathrm{kg} / \mathrm{m}^{2}\right)$ & $25.0(3.2)$ & $25.1(3.0)$ & $25.5(3.4)$ \\
\hline
\end{tabular}

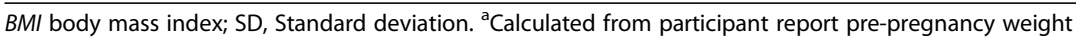




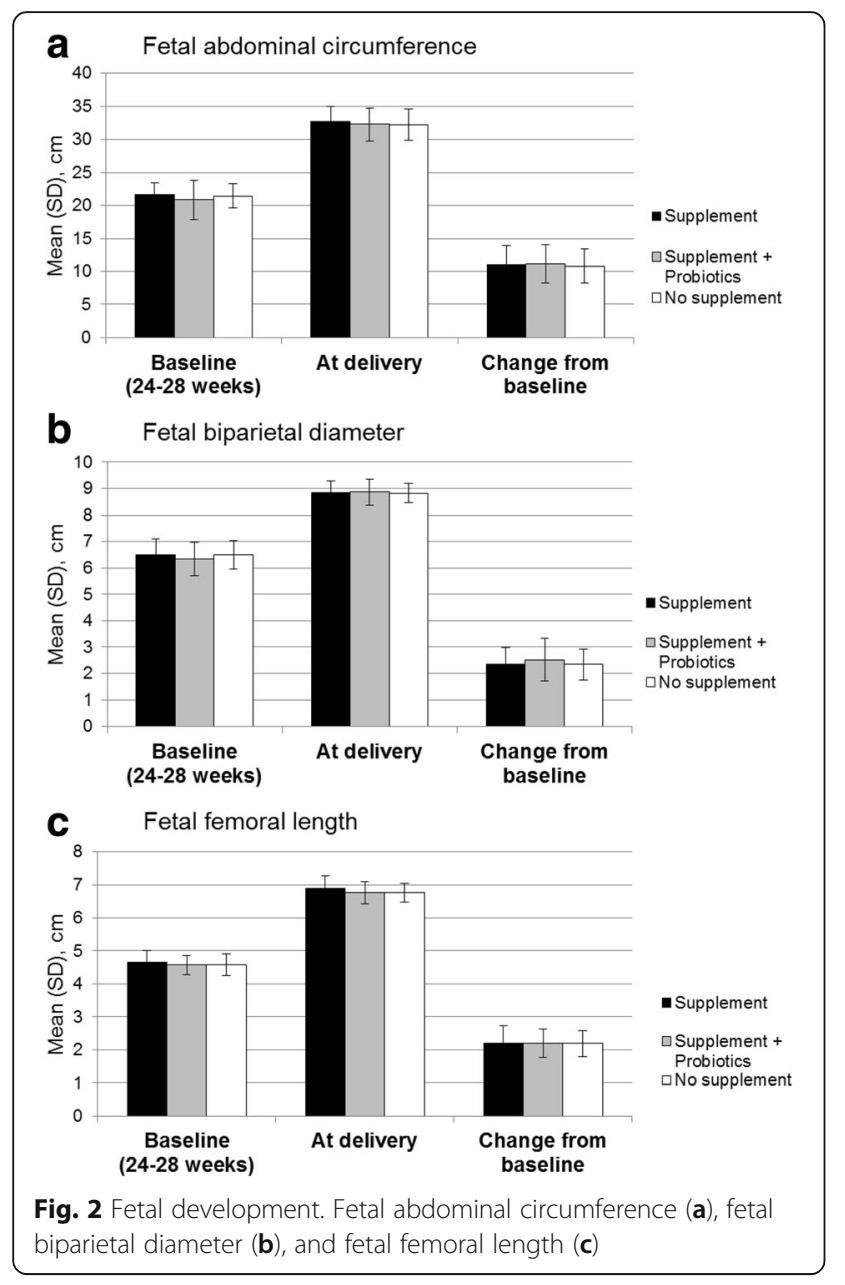

supplement was $0.82(0.40-1.68)$ compared to no supplement.

Adverse events in infants Overall, infant morbidity was similar among groups. However, there was a trend towards more serious gastrointestinal infections in the no supplement group (10.3\%) than in the combined supplemented groups (4.3\%). Moreover, we noticed significantly less frequent vomiting cases in the combined supplemented group compared to no supplement group (0.0\% vs. $5.9 \%$, $p=0.011)$. In total, there were four infant deaths. Two deaths were reported in the supplement group, one in the supplement plus probiotics group, and one in the no supplement group (Table 5). None of these cases were considered related to product consumption in the medical records.

Changes in anthropometric measurements over time In the three study groups, mean weight-for-age, length-for-age, BMI-for-age and head circumference-for-age z-scores were below the WHO median values. At birth, all anthropometric measurements were similar between the groups. Interestingly, at 2 months of age, i.e. at the end of the mandatory intake of maternal supplementation and breastfeeding, the combined supplemented group had higher weight-for-age $(-0.40,95 \% \mathrm{CI}:-0.54$ to -0.25 vs. $-0.57,95 \%$ CI: -0.77 to -0.36$)$, length-for-age $(-0.37,95 \% \mathrm{CI}:-0.55$ to -0.19 vs. $-0.51,95 \%$ CI: -0.77 to -0.25$)$, and BMI-for-age $(-0.25,95 \% \mathrm{CI}:-0.43$ to -0.07 vs. -0.39 , 95\% CI: -0.65 to -0.14$) \mathrm{z}$-scores than the no supplement group (Fig. 3a, b, c, ). However, these differences did not reach significance. This trend persisted throughout the study follow-up period, up to 12 months. At 12 months of age, the combined supplemented groups gained more weight (adjusted mean in kg, 95\% CI: 8.97 [8.84-9.09] vs. 8.61 [8.43-8.78]; $p=0.001$ ) and height (adjusted mean in cm, 95\% CI: 74.2 [73.8-74.6] vs. 73.4 [72.8-74.0]; $p=0.031)$ compared to the no supplement group (Table 6) and had a significantly higher weight-for-age $\mathrm{z}$-score (-0.88 vs. $-0.62, p=0.045$ ) (Fig. 3a). There was neither statistically significant difference nor trend for a higher head circumference-for-age $\mathrm{z}$-score in the combined versus no supplement groups throughout the study (Fig. 3d). No statistically significant differences on growth outcome per gender were observed between groups (data not shown).

\section{Discussion}

In the present study, we assessed the effect of an oral maternal nutritional supplement formulated with the

Table 4 Pregnancy-related serious adverse events (SAEs)

\begin{tabular}{|c|c|c|c|c|c|c|c|}
\hline \multirow[b]{2}{*}{ Pregnancy, puerperium and perinatal conditions } & \multicolumn{2}{|c|}{$\begin{array}{l}\text { Supplement } \\
\qquad(n=70)\end{array}$} & \multicolumn{2}{|c|}{$\begin{array}{l}\text { Supplement + probiotics } \\
\qquad(n=70)\end{array}$} & \multicolumn{2}{|c|}{$\begin{array}{l}\text { No supplement } \\
\quad(n=68)\end{array}$} & \multirow[b]{2}{*}{$p$-value } \\
\hline & \# Events & $\begin{array}{l}\text { \# Mothers } \\
(\%)\end{array}$ & \# Events & $\begin{array}{l}\text { \# Mothers } \\
(\%)\end{array}$ & \# Events & $\begin{array}{l}\text { \# Mothers } \\
(\%)\end{array}$ & \\
\hline Fetal growth restriction & 0 & $0(0.0 \%)$ & 0 & $0(0.0 \%)$ & 1 & $1(1.5 \%)$ & - \\
\hline Gestational hypertension & 1 & $1(1.4 \%)$ & 1 & $1(1.4 \%)$ & 0 & $0(0.0 \%)$ & 1.000 \\
\hline Pre-eclampsia & 1 & $1(1.4 \%)$ & 1 & $1(1.4 \%)$ & 1 & $1(1.5 \%)$ & 1.000 \\
\hline Premature labor & 3 & $3(4.3 \%)$ & 1 & $1(1.4 \%)$ & 1 & $1(1.5 \%)$ & 0.620 \\
\hline Genito-urinary tract infections & 15 & $14(20.0 \%)$ & 9 & $7(10.0 \%)$ & 3 & $3(4.4 \%)$ & 0.154 \\
\hline Tolerance: nausea, vomiting and diarrhea & 6 & $4(5.7 \%)$ & 11 & 7 (10.0\%) & 1 & 1 (1.5\%) & 0.532 \\
\hline
\end{tabular}

Data are number (\%) of mothers with at least one event; ${ }^{*}$ Supplement + probiotics versus Supplement 
Table 5 Adverse events in infants (combined supplemented groups versus non-supplemented group)

\begin{tabular}{|c|c|c|c|c|c|}
\hline & \multicolumn{2}{|c|}{$\begin{array}{c}\text { Combined }^{\S} \\
(n=140)\end{array}$} & \multicolumn{2}{|c|}{$\begin{array}{l}\text { No Supplement } \\
\quad(n=68)\end{array}$} & \multirow[t]{2}{*}{$\begin{array}{l}p \text {-value* Combined vs. } \\
\text { No supplement }\end{array}$} \\
\hline & \# Events & $\begin{array}{c}\text { \# Infants } \\
(\%)\end{array}$ & \# Events & $\begin{array}{c}\# \text { Infants } \\
(\%)\end{array}$ & \\
\hline \multicolumn{6}{|l|}{ Adverse events of diarrhea } \\
\hline Serious diarrhea & $1^{* *}$ & $1(0.7 \%)$ & 0 & $0(0.0 \%)$ & 1.000 \\
\hline Diarrhea & 21 & $19(13.6 \%)$ & 12 & $11(16.2 \%)$ & 0.675 \\
\hline Total & 22 & 20 & 12 & 11 & - \\
\hline \multicolumn{6}{|l|}{ Other serious adverse events of interest } \\
\hline All respiratory infections/symptoms & 309 & $119(85.0 \%)$ & 177 & $63(92.6 \%)$ & 0.179 \\
\hline Gastrointestinal infections & 7 & $6(4.3 \%)$ & 7 & $7(10.3 \%)$ & 0.126 \\
\hline Allergies & 11 & $7(5.0 \%)$ & 4 & $3(4.4 \%)$ & 1.000 \\
\hline Death $^{a}$ & 3 & $3(2.4 \%)$ & 1 & $1(1,5 \%)$ & \\
\hline \multicolumn{6}{|l|}{ Other adverse events of interest } \\
\hline Vomiting & 0 & $0(0.0 \%)$ & 5 & $4(5.9 \%)$ & 0.011 \\
\hline
\end{tabular}

Data are number of infants with at least one event (\%). ${ }^{\S}$ Combined: supplement and supplement + probiotics groups. ${ }^{*}$ Exact Fisher test $p$-value. ${ }^{* *}$ Event reported in the supplement group. ${ }^{a}$ As confirmed by clinicians, not related to product consumption

probiotics Lactobacillus rhamnosus and Bifidobacterium lactis on the incidence of diarrhea in infants from birth to one year. The supplement was consumed by expecting mothers during the third trimester of pregnancy and continued for at least two months post-partum while breastfeeding. There was no significant difference in the incidence of infant diarrhea between treatment groups. The incidence of diarrhea was also similar between the supplemented groups and the no supplement group. In a clinical trial setting, an overall lower incidence of infant diarrhea is often observed as compared to field data records. It is likely that mothers involved in clinical trials provide more care and attention to their infants than during everyday life when infants do not undergo such a constant growth and health monitoring. Nevertheless, in the present study the incidence rate of infant diarrhea was not dramatically lower than the estimates from the two reference articles used for sample size calculation [24, 25]. Even if the supplement plus probiotics group pointed towards a slight numerical trend compared to the supplement and no supplement groups, the expected 50\% reduction in incidence of infant diarrhea was not achieved in the current clinical trial setting. In order to reach statistical significance more than 300 infants per group would have been required. In this context, one could consider that the study was underpowered to show such a dramatic impact on incidence of infant diarrhea.

Secondary endpoints were evaluated as planned, and an additional post-hoc analysis was performed. It compared the combined groups receiving oral supplements (with and without probiotics) with the no supplement group to evaluate the effect of maternal supplementation on maternal health and fetal and infant growth. Some studies have described the safety and effect of a nutritional supplement containing a mix of probiotics for pregnant and breastfeeding women on fetus and infant [12-15], but none have fully examined the impact of oral nutritional supplements on fetal and infant growth. Our study provides preliminary evidence suggesting a beneficial effect of oral nutritional supplements, with and without probiotics, during the third trimester of pregnancy on optimal maternal weight gain and infant growth at one year.

Oral maternal nutritional supplements are formulated to provide a balance of energy, protein and nutrients to support a healthy pregnancy. However the consumption of too many calories during pregnancy may increase a woman's risk for excessive weight gain which has been linked with gestational diabetes [26, 27] and hypertension [26], cesarean birth [26, 28], macrosomia [28-30] and large-for-gestational age infants [26, 28], later type 2 diabetes [31] and obesity [26], along with childhood obesity [26]. During the third trimester of pregnancy, our study showed that maternal weight gain among the three study groups was equivalent despite an increase in calorie intake of $280 \mathrm{kcal}$ per day in the supplemented groups. This is consistent with a recent report indicating a reduced occurrence of central adiposity (i.e. waist circumference $\geq 80 \mathrm{~cm}$ ) at 6 months after birth when probiotics are given to pregnant women in combination with dietary counseling [32]. Moreover, our results are in agreement with the findings of Luoto et al. who reported a reduction of gestational diabetes in mothers fed probiotics Lactobacillus rhamnosus and Bifidobacterium lactis together with dietary counseling from the first trimester of pregnancy and adequate prenatal and postnatal growth rates (body weight and length) of infants during the first two years of life [33]. Additionally, our study showed no differences in the incidence of pregnancy-related AEs and 


\section{a Weight-for-age z-score over time}

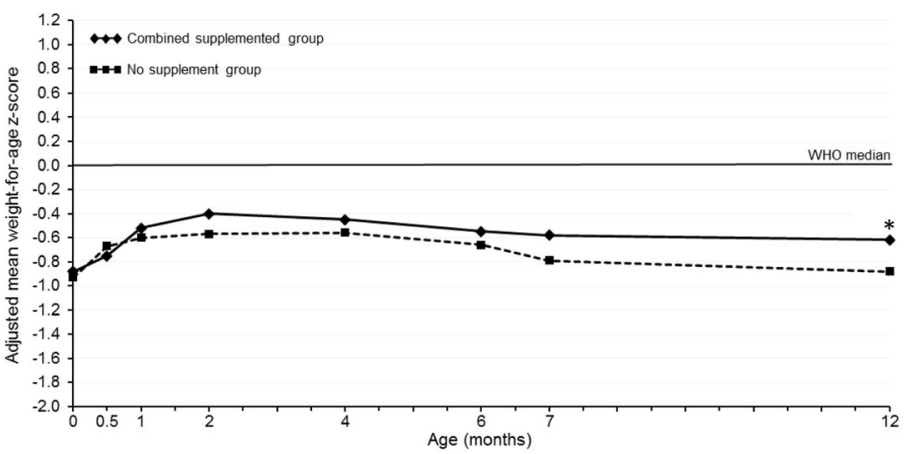

b Length-for-age z-score over time

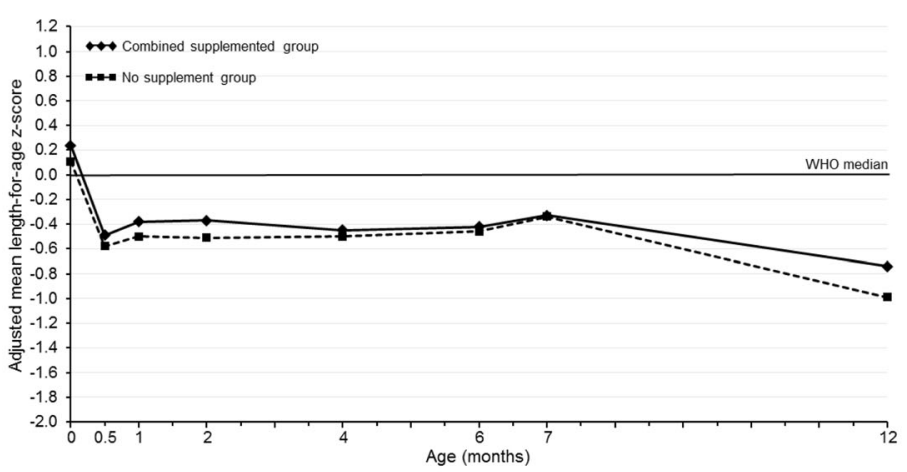

C BMl-for-age z-score over time

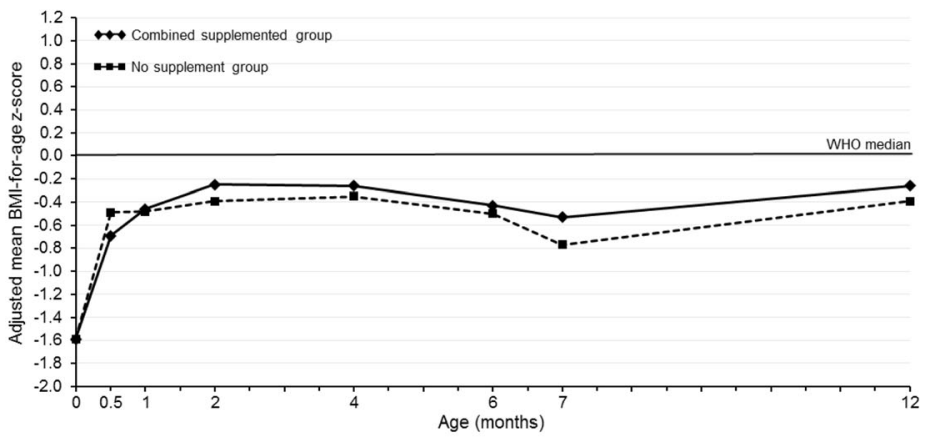

d Head circumference-for-age z-score over time

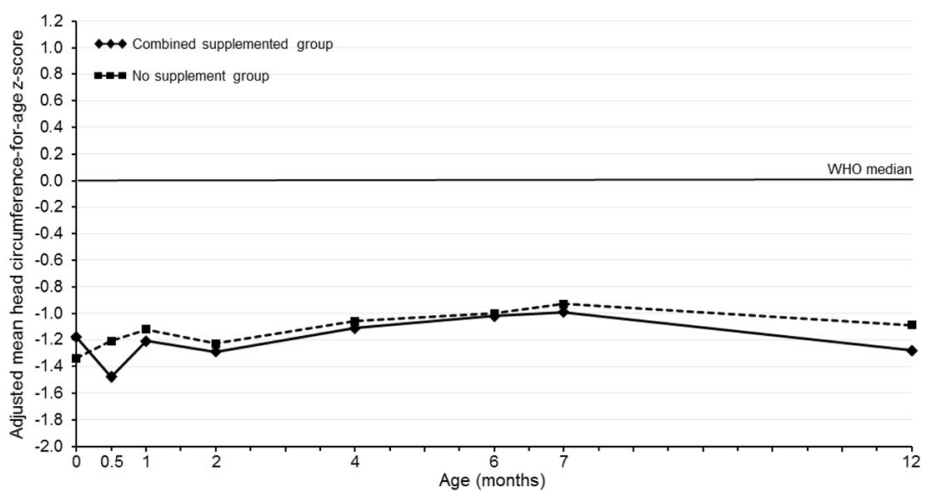

Fig. 3 Infant anthropometrics over the study period. Weight-for-age (a), length-for-age (b), BMl-for-age (c) and head circumference-for-age (d) $z$-scores (World Health Organization growth reference). ${ }^{*} p=0.045$ 
Table 6 Infant anthropometrics at 12 months of age

\begin{tabular}{lcccccc}
\hline $\begin{array}{l}\text { Measurement, adjusted } \\
\text { mean }(95 \% \mathrm{Cl})\end{array}$ & $\begin{array}{c}\text { Supplement } \\
(n=62)\end{array}$ & $\begin{array}{c}\text { Supplement }+ \\
\text { probiotics }(n=60)\end{array}$ & $\begin{array}{c}\text { Combined }^{\mathrm{a}} \\
(n=122)\end{array}$ & $\begin{array}{c}\text { No supplement } \\
(n=61)\end{array}$ & $\begin{array}{c}p \text {-value } \\
\begin{array}{l}\text { Supplement + probiotics } \\
\text { vs. supplement }\end{array}\end{array}$ & $\begin{array}{c}\text { Combined vs. } \\
\text { No supplement }\end{array}$ \\
\hline Weight $(\mathrm{kg})$ & $8.92(8.75-9.09)$ & $9.06(8.88-9.24)$ & $8.97(8.84-9.09)$ & $8.61(8.43-8.78)$ & 0.273 & 0.001 \\
Length $(\mathrm{cm})$ & $73.8(73.3-74.4)$ & $74.6(74.1-75.2)$ & $74.2(73.8-74.6)$ & $73.4(72.8-74.0)$ & 0.057 & 0.031 \\
Head circumference $(\mathrm{cm})$ & $44.0(43.7-44.3)$ & $44.1(43.9-44.5)$ & $44.1(43.9-44.3)$ & $44.2(44.0-44.7)$ & 0.413 & 0.317 \\
Body mass index $\left(\mathrm{kg} / \mathrm{m}^{2}\right)$ & $16.3(16.0-16.7)$ & $16.1(15.8-16.5)$ & $16.2(16.0-16.5)$ & $16.0(15.7-16.4)$ & 0.496 & 0.371 \\
\hline
\end{tabular}

Analyzed by ANCOVA, correcting for sex and available baseline; ${ }^{a}$ Combined: supplement and supplement + probiotics groups; $\mathrm{Cl}$ confidence interval

SAEs, and adverse fetal outcomes between all study groups. Overall, the optimal maternal weight gain and positive tolerance data indicate that maternal feeding with a supplement containing or not probiotics during the last trimester of pregnancy is safe for the mother and the fetus with respect to its energy density and nutritive value.

We further explored the effect of the supplementation compared to the current nutrition practice, i.e. no supplement. The two supplemented groups were thus subsequently pooled and compared to the no supplement group in a post-hoc analysis. No difference was observed between the two groups with respect to AEs or SAEs in infants, except for vomiting which appeared significantly less frequent in the combined supplemented group than in the no supplement group. Moreover, a trend for less serious gastrointestinal infections in the combined supplemented group was noticed. Altogether, these observations are suggestive of the potential beneficial effect of the maternal supplement on infant health, regardless of the addition of probiotics. Additional studies would be needed to confirm these observations.

As giving nutritional supplements to breastfeeding mothers in developing countries could presumably improve infant growth, in the post-hoc analysis, we also examined the effect of the combined supplemented group on infant growth. In our study, all infant growth parameters were below WHO median values, at all study time points (up to 12 months of age) and in both the combined supplemented and the no supplement groups. This finding is consistent with the results from the Food and Nutrition Research Institute of the Department of Science and Technology, 8th National Nutrition Survey showing an estimated $19.9 \%$ of Filipino children aged 0 to 5 years are underweight [34]. Nevertheless, it should be noted that the growth curves of the combined supplemented group was higher (except for head circumference) compared with the no supplement group for all time points.

Starting around 6-7 months of age, a negative break in the infant growth curves was observed which probably corresponds to the start of the weaning period. At this time, complementary foods are introduced and the infant is at an increased risk of being exposed to food-borne illness. Furthermore, complementary foods may often be of lower nutritional value than infant formula, resulting in the consumption of a non-optimal amount of calories, protein and essential micronutrients. In the Philippines, among the lower socio economic population, an increased risk of infection combined with inadequate nutrition may be contributing to the decline in infant growth rate. This could explain what was observed at 6 months of age.

At 12 months of age, infants in the combined supplemented group had gained significantly more weight than the infants in the no supplement group. This suggests a potential long-term beneficial effect of the supplementation. The GUSTO (Growing Up in Singapore Towards healthy Outcomes) study group recently showed that food consumption was altered during pregnancy and the postpartum period in a large number of women from a multiethnic Asian population who were heavily influenced by traditional beliefs surrounding these periods [35]. This study group further investigated the association between maternal macronutrient intake during pregnancy and infant birth size in the same Asian population [36]. Similar to what was observed in the no supplement group of our study, the study concluded that maternal macronutrient intake was not associated with infant birth weight [36]. Additionally, Bhargava examined the effect of dietary intake on growth and morbidity in Filipino infants from 2 to 24 months and reported calcium, protein to energy intake ratio, and $\beta$-carotene were significantly correlated with height, weight, and morbidity indexes, respectively [37]. In western infants, clinically significant catch-up growth (defined as a gain in weight $\mathrm{z}$-scores $>0.67$ ) between 0 and 24 months was associated with greater weight, height, BMI and body fat at five years compared to similar aged children without catch-up growth [38]. In this study, the mean product effect on weight-for-age z-scores was only equal to 0.211 . Nevertheless, there is a need for a better understanding of the benefits and risks of greater weight gain to promote improved health outcomes and the later risk of adiposity and obesity [39]. Further investigating of these potential determinants of excess infant adiposity gain could lead to intervention strategies in clinical and public health settings to prevent childhood obesity and its consequences. 
We acknowledge some limitations in our study. First, supplement consumption was not monitored beyond the 2-month postnatal period, and the impact of continued supplement consumption on breast-fed infant growth was not examined. Second, the potential beneficial effect of the supplements on breast milk composition was not studied and compared between groups. This could have provided insights on the effect observed on infant growth. Third, the absence of increased weight gain in pregnant women taking the supplement (with or without probiotics) cannot be generalized to the whole population as this observation may be linked to a genetic component specific to this sample of Filipino women. Indeed, women's weight in Western countries is generally higher and women tend to gain more weight during pregnancy (for women with a normal weight, i.e. a BMI of 18.5-24.9, the recommended range of total weight gain during pregnancy is $11-16 \mathrm{~kg}$ [40]). Moreover, the mother's diet and exercise during the third trimester of pregnancy and the 2-month postnatal period was not taken into account, as these data were not recorded during the trial. However, considering that all recruited mothers had comparable socioeconomic profiles and came from the same geographical area, one can assume that their global calorie intake was similar. Nevertheless, a better monitoring of mothers' diet and exercise should be included in future nutritional intervention trials. Finally, limitations also include the study population of women in the third trimester of pregnancy living in the greater Manila region, limiting the generalizability of these findings to all stages of pregnancy and all regions of the Philippines.

\section{Conclusion}

Oral maternal nutritional supplements formulated with or without Lactobacillus rhamnosus and Bifidobacterium lactis during the last trimester of pregnancy and through the first two months of exclusive breastfeeding were well tolerated and safe for mothers, fetus and infants. Even though in the present study no difference in incidence of infant diarrhea was observed between the three groups, the analysis of combined supplemented groups showed beneficial effects of maternal supplementation on infant weight and length gains at 12 months of age.

\section{Abbreviations}

AEs: Adverse events; BMI: Body mass index; CFU: Colony forming units; Cl: Confidence interval; FAS: Full analysis set; GI: Gastro-intestinal; HIV: human immunodeficiency virus; SAEs: Serious adverse events; SD: Standard deviations; WHO: World Health Organization; WHO-ART: WHO adverse reactions terminology

\section{Acknowledgements}

The authors thank Dr. Philippe Steenhout for assistance with the study design and helpful scientific discussions. The authors also thank Julie Chenal for medical writing services and Emilie Perrin and Shahram Emady-Azar for their support with the statistical analysis.
Funding

Nestec Ltd. sponsored the study.

Availability of data and materials

The study protocol as well as the datasets used and/or analyzed during the current study are available from the corresponding author on reasonable request.

Authors' contributions

VG, JM, and JB conceived and designed the study. JB, KV, SP and SV developed the statistical analysis plan. VG, JM, SP, JB, KV and SV jointly developed the structure and the arguments for the paper. RD performed clinical samples laboratory analyses. All contributed to the writing of the manuscript. All agreed with the manuscript results and conclusion. All made critical revisions and approved final version. All authors reviewed and approved the final manuscript.

\section{Ethics approval and consent to participate}

The study was performed in accordance with the Helsinki Declaration and complied with Good Clinical Practices as laid out in the International Conference on Harmonization guidelines. It was approved by the institution's Ethics Committee (University of the Philippines Manila - National Institutes of Health Ethics Review Board; protocol number NIH 2009-027). Participants provided written informed consent to share data and participation was voluntary.

\section{Competing interests}

VG and JM received a study grant from Nestlé Nutrition for this study. SP, JB, KV and SV are Nestlé employees.

\section{Publisher's Note}

Springer Nature remains neutral with regard to jurisdictional claims in published maps and institutional affiliations.

\section{Author details}

'Department of Pediatrics, Philippines General Hospital, City, Manila, Philippines. ${ }^{2}$ Institute of Nutritional Science, Nestlé Research Center, Vers-chez-les-Blanc, 1000, 26 Lausanne, Switzerland. ${ }^{3}$ Internal Medicine \& Infectious Diseases, Philippines General Hospital, Manila, Philippines. ${ }^{4}$ Clinical Research, Nestlé Nutrition, Vevey, Switzerland. ${ }^{5}$ Janssen R\&D LLC, Welsh \& McKean Roads, Springhouse, PA 19477, USA. ${ }^{6}$ Department of Obstetrics \& Gynecology, Philippines General Hospital, Manila, Philippines.

Received: 21 December 2017 Accepted: 15 May 2018

Published online: 31 May 2018

References

1. Ramakrishnan U, Grant F, Goldenberg T, Zongrone A, Martorell R. Effect of women's nutrition before and during early pregnancy on maternal and infant outcomes: a systematic review. Paediatr Perinat Epidemiol. 2012; 26(Suppl 1):285-301.

2. Ramakrishnan U, Imhoff-Kunsch B, Martorell R. Maternal nutrition interventions to improve maternal, newborn, and child health outcomes. Nestlé Nutrition Institute workshop series. 2014;78:71-80.

3. Milman N, Paszkowski T, Cetin I, Castelo-Branco C. Supplementation during pregnancy: beliefs and science. Gynecological endocrinology : the official journal of the International Society of Gynecological Endocrinology. 2016:1-8.

4. Belkacemi L, Nelson DM, Desai M, Ross MG. Maternal undernutrition influences placental-fetal development. Biol Reprod. 2010;83(3):325-31.

5. Nnam NM. Improving maternal nutrition for better pregnancy outcomes. Proc Nutr Soc. 2015;74(4):454-9.

6. Bhutta ZA, Das JK, Rizvi A, Gaffey MF, Walker N, Horton S, Webb P, Lartey A, Black RE, Lancet nutrition interventions review $G$, et al. Evidence-based interventions for improvement of maternal and child nutrition: what can be done and at what cost? Lancet. 2013:382(9890):452-77.

7. Singhal A, Lucas A. Early origins of cardiovascular disease: is there a unifying hypothesis? Lancet. 2004;363(9421):1642-5.

8. Yang Z, Huffman SL. Nutrition in pregnancy and early childhood and associations with obesity in developing countries. Maternal \& child nutrition. 2013;9(Suppl 1):105-19.

9. Haider BA, Bhutta ZA. Multiple-micronutrient supplementation for women during pregnancy. Cochrane Database Syst Rev. 2015;11:CD004905. 
10. Gomez Arango LF, Barrett HL, Callaway LK, Nitert MD. Probiotics and pregnancy. Current diabetes reports. 2015;15(1):567.

11. Hashemi A, Villa CR, Comelli EM. Probiotics in early life: a preventative and treatment approach. Food Funct. 2016;7(4):1752-68.

12. Rautava S, Kalliomäki M, Isolauri E. Probiotics during pregnancy and breastfeeding might confer immunomodulatory protection against atopic disease in the infant. J Allergy Clin Immunol. 2002;109(1):119-21.

13. Kalliomäki M, Salminen S, Poussa T, Isolauri E. Probiotics during the first 7 years of life: a cumulative risk reduction of eczema in a randomized, placebo-controlled trial. J Allergy Clin Immunol. 2007;119(4):1019-21.

14. Lahtinen SJ, Boyle RJ, Kivivuori S, Oppedisano F, Smith KR, Robins-Browne R, Salminen SJ, Tang ML. Prenatal probiotic administration can influence Bifidobacterium microbiota development in infants at high risk of allergy. J Allergy Clin Immunol. 2009;123(2):499-501. e498

15. Gueimonde M, Sakata S, Kalliomäki M, Isolauri E, Benno Y, Salminen S. Effect of maternal consumption of lactobacillus GG on transfer and establishment of fecal bifidobacterial microbiota in neonates. J Pediatr Gastroenterol Nutr. 2006;42(2):166-70.

16. Allen SJ, Okoko B, Martinez E, Gregorio G, Dans LF. Probiotics for treating infectious diarrhoea. The Cochrane database of systematic reviews. 2004;2: CD003048.

17. Bergonzelli GE, Blum S, Brussow H, Corthesy-Theulaz I. Probiotics as a treatment strategy for gastrointestinal diseases? Digestion. 2005;72(1):57-68.

18. Weizman Z, Asli G, Alsheikh A. Effect of a probiotic infant formula on infections in child care centers: comparison of two probiotic agents. Pediatrics. 2005;115(1):5-9.

19. Chouraqui JP, Van Egroo LD, Fichot MC. Acidified milk formula supplemented with bifidobacterium lactis: impact on infant diarrhea in residential care settings. J Pediatr Gastroenterol Nutr. 2004;38(3):288-92.

20. Saavedra JM, Bauman NA, Oung I, Perman JA, Yolken RH. Feeding of Bifidobacterium bifidum and Streptococcus thermophilus to infants in hospital for prevention of diarrhoea and shedding of rotavirus. Lancet. 1994; 344(8929):1046-9.

21. Guandalini S, Pensabene L, Zikri MA, Dias JA, Casali LG, Hoekstra H, Kolacek S, Massar K, MiceticTurk D, Papadopoulou A. lactobacillus GG administered in oral rehydration solution to children with acute diarrhea: a multicenter European trial. J Pediatr Gastroenterol Nutr. 2000:30(1):54-60.

22. Canani RB, Cirillo P, Terrin G, Cesarano L, Spagnuolo MI, De Vincenzo A, Albano F, Passariello A, De Marco G, Manguso F, et al. Probiotics for treatment of acute diarrhoea in children: randomised clinical trial of five different preparations. BMJ (Clinical research ed). 2007:335(7615):340.

23. Corrêa NB, Péret Filho LA, Penna FJ, Lima FMS, Nicoli JR. A randomized formula controlled trial of Bifidobacterium lactis and Streptococcus thermophilus for prevention of antibiotic-associated diarrhea in infants. $J$ Clin Gastroenterol. 2005;39(5):385-9.

24. Hengstermann S, Mantaring JB 3rd, Sobel HL, Borja VE, Basilio J, lellamo AD, Nyunt US. Formula feeding is associated with increased hospital admissions due to infections among infants younger than 6 months in manila, Philippines. J Hum Lact. 2010;26(1):19-25.

25. Ortiz-Andrellucchi A, Sánchez-Villegas A, Rodríguez-Gallego C, Lemes A, Molero T, Soria A, Pena-Quintana L, Santana M, Ramírez O, García J. Immunomodulatory effects of the intake of fermented milk with lactobacillus casei DN114001 in lactating mothers and their children. Br J Nutr. 2008;100(4):834-45

26. Gaillard R, Durmuş B, Hofman A, Mackenbach JP, Steegers EA, Jaddoe WW. Risk factors and outcomes of maternal obesity and excessive weight gain during pregnancy. Obesity. 2013;21(5):1046-55.

27. Leng J, Shao P, Zhang C, Tian H, Zhang F, Zhang S, Dong L, Li L, Yu Z, Chan $J C$. Prevalence of gestational diabetes mellitus and its risk factors in Chinese pregnant women: a prospective population-based study in Tianjin, China. PLoS One. 2015;10(3):e0121029.

28. Stotland NE, Hopkins LM, Caughey AB. Gestational weight gain, macrosomia, and risk of cesarean birth in nondiabetic nulliparas. Obstet Gynecol. 2004;104(4):671-7.

29. Ludwig DS, Currie J. The association between pregnancy weight gain and birthweight: a within-family comparison. Lancet. 2010;376(9745):984-90.

30. Viswanathan M, Siega-Riz AM, Moos M-K, Deierlein A, Mumford S, Knaack J, Thieda P, Lux LJ, Lohr KN. Outcomes of maternal weight gain, Evidence Report//echnology Assessment No. 168. (Prepared by RTI InternationalUniversity of North Carolina Evidence-based Practice Center under Contract No. 290-02-0016.) AHRQ Publication No. 08-E009. Rockville, MD: Agency for Healthcare Research and Quality; 2008.
31. Al Mamun A, Mannan M, O'Callaghan MJ, Williams GM, Najman JM, Callaway LK. Association between gestational weight gain and postpartum diabetes: evidence from a community based large cohort study. PLoS One. 2013;8(12):e75679.

32. IImonen J, Isolauri E, Poussa T, Laitinen K. Impact of dietary counselling and probiotic intervention on maternal anthropometric measurements during and after pregnancy: a randomized placebo-controlled trial. Clin Nutr. 2011; 30(2):156-64.

33. Luoto R, Laitinen $\mathrm{K}$, Nermes M, Isolauri E. Impact of maternal probioticsupplemented dietary counselling on pregnancy outcome and prenatal and postnatal growth: a double-blind, placebo-controlled study. Br J Nutr. 2010; 103(12):1792-9.

34. The Food and Nutrition Research Institute of the Department of Science and Technology. 8th National Nutrition Survey. Manila, Philippines; 2013. [http://www.fnri.dost.gov.ph/index.php/nutrition-statistic/19-nutritionstatistic/118-8th-national-nutrition-survey. Accessed 05 Feb.2017].

35. Chen LW, Low YL, Fok D, Han WM, Chong YS, Gluckman P, Godfrey K, Kwek $\mathrm{K}$, Saw SM, Soh SE, et al. Dietary changes during pregnancy and the postpartum period in Singaporean Chinese, Malay and Indian women: the GUSTO birth cohort study. Public Health Nutr. 2014;17(9):1930-8.

36. Chong MF, Chia AR, Colega M, Tint MT, Aris IM, Chong YS, Gluckman P, Godfrey KM, Kwek K, Saw SM, et al. Maternal protein intake during pregnancy is not associated with offspring birth weight in a multiethnic Asian population. J Nutr. 2015;145(6):1303-10.

37. Bhargava A. Protein and micronutrient intakes are associated with child growth and morbidity from infancy to adulthood in the Philippines. J Nutr. 2016;146(1):133-41.

38. Ong KK, Ahmed ML, Emmett PM, Preece MA, Dunger DB. Association between postnatal catch-up growth and obesity in childhood: prospective cohort study. Bmj. 2000;320(7240):967-71.

39. Taveras EM, Rifas-Shiman SL, Sherry B, Oken E, Haines J, Kleinman K, RichEdwards JW, Gillman MW. Crossing growth percentiles in infancy and risk of obesity in childhood. Archives of pediatrics \& adolescent medicine. 2011; 165(11):993-8.

40. ACOG. Committee opinion no. 548: weight gain during pregnancy. Obstet Gynecol. 2013;121(1):210-2.

\section{Ready to submit your research? Choose BMC and benefit from:}

- fast, convenient online submission

- thorough peer review by experienced researchers in your field

- rapid publication on acceptance

- support for research data, including large and complex data types

- gold Open Access which fosters wider collaboration and increased citations

- maximum visibility for your research: over $100 \mathrm{M}$ website views per year

At BMC, research is always in progress.

Learn more biomedcentral.com/submissions 